\title{
Band gap opening in graphene: a short theoretical study
}

\author{
Sivabrata Sahu' ${ }^{1}$ G. C. Rout $^{2}$
}

Received: 19 March 2016/ Accepted: 13 February 2017/Published online: 8 March 2017

(C) The Author(s) 2017. This article is published with open access at Springerlink.com

\begin{abstract}
Graphene, being a gapless semiconductor, cannot be used in pristine form for nano-electronic applications. Therefore, it is essential to generate a finite gap in the energy dispersion at Dirac point. We present here the tightbinding model Hamiltonian taking into account of various interactions for tuning band gap in graphene. The model Hamiltonian describes the hopping of the $\pi$-electrons up to third nearest-neighbours, substrate effects, Coulomb interaction at two sub-lattices, electron-phonon interaction in graphene-on-substrates and high phonon frequency vibrations, besides the bi-layer graphene. We have solved the Hamiltonian using Zubarev's double time single particle Green's function technique. The quasi-particle energies, electron band dispersions, the expression for effective band gap and the density of states (DOS) are calculated numerically. The results are discussed by varying different model parameters of the system. It is observed that the electron DOS and band dispersion exhibit linear energy dependence near Dirac point for nearest-neighbour hopping integral. However, the second and third nearest-neighbour hoppings provide asymmetry in DOS. The band dispersions exhibit wider band gaps with stronger substrate effect. The modified gap in graphene-on-substrate attains its maximum value for Coulomb interaction energy $U_{\mathrm{C}}=1.7 t_{1}$. The
\end{abstract}

G. C. Rout

gcr@iopb.res.in

Sivabrata Sahu

siva1987@iopb.res.in

1 School of Applied Sciences (Physics), KIIT University, Bhubaneswar, Odisha 751024, India

2 Condensed Matter Physics Group, Physics Enclave, Plot No664/4825, Lane-4A, Shree Vihar, Bhubaneswar, Odisha 751031, India critical Coulomb interaction is enhanced to $U_{\mathrm{C}}=2.5 t_{1}$ to produce maximum band gap in the presence of electronphonon interaction and phonon vibration. The bi-layer graphene exhibits Mexican hat type band gap near Dirac point for transverse gating potential. The other conclusions for the present work are described in the text.

Keywords Graphene · Coulomb interaction · Electronphonon interaction $\cdot$ Bi-layer graphene

\section{Introduction}

Graphene is one-atom-thick two-dimensional structure with carbon atoms packed in a honeycomb lattice. Its recent experimental discovery has stimulated extensive investigations on every aspect of this novel material $[1,2]$. The tightbinding calculation for graphene shows that its conduction and valence bands touch at six Dirac points in the Brillouin zone [3] where energy dispersions are linear with respect to momentum. This unique band dispersion in graphene leads to graphene's novel physical and electronic properties such as room temperature quantum Hall effects and high charge carrier mobility [4-6]. Graphene, being a gapless semimetal, cannot be used in pristine form for nano-electronic applications. Therefore, it is necessary to open a finite gap in the energy dispersions at $\mathrm{K}$ point by various mechanisms $[7,8]$. When graphene lies on born nitride $(\mathrm{BN})$ substrate, a small gap $(\sim 100 \mathrm{meV})$ is observed $[9,10]$. Similarly, a band gap of $250 \mathrm{meV}$ is observed for silicon carbide substrate $[11,12]$. There is a great effort for producing a gap $(\sim 1 \mathrm{eV})$ which is observed in germanium/silicon for the application in digital electronics.

The recent work indicates that the carbon layer is cocovalently bonded to the SiC sub-lattice [13-17]. The band 
gap opening for graphene-on-ruthenium metal also arises due to symmetry breaking in the system [18, 19]. McCann and Falko $[8,20]$ have proposed that bi-layer graphene can develop a gap, when transverse electric field is applied between two surfaces of the system acting as a gating. Band gap in bi-layer graphene has been observed experimentally by infrared spectroscopy [21, 22] and angle resolved photo emission spectroscopy (ARPES) [23]. Hague [24, 25] has proposed a theoretical model calculation taking attractive interaction modified through phonons in polarisable substrate for the strong enhancement of the band gap in graphene substrates. The band gap of several orders in eV can be prepared in Graphane [26] and Fluorographene $[27,28]$ by chemical modification of the system with hydrogen and fluorine, respectively. Atomic thick boron nitride $(\mathrm{BN})$ forms a honeycomb lattice where the $\pi$ orbitals on $\mathrm{N}$ sites are shifted up in energy by $+\Delta$ and decreased in energy of $-\Delta$ on B site causing a gap of $2 \Delta$ [29]. The band gap of $5.56 \mathrm{eV}$ is observed experimentally on monolayer BN systems.

The role of Coulomb interaction in low-dimensional systems provides unique opportunity for theoretical as well as experimental studies. The graphene [30,31], varieties of semiconductor surfaces like $\mathrm{Si}, \mathrm{Ce}, \mathrm{Sn}, \mathrm{Pb}$ [32], Bechgard salt [33] and doped polymers [34, 35] display strong on-site as well as inter-site Coulomb interactions. It is observed that on-site Coulomb interaction in graphene is $U=3.3 t_{1}$ and the nearest-neighbour Coulomb interaction is $V=$ $2.0 t_{1}$ where the nearest-neighbour hopping integral is $t_{1}=2.8 \mathrm{eV}$ [36]. It has been reported that the effective Hubbard interaction is $U=3.3 t_{1}$ in the close vicinity of the separation between conducting graphene and insulating phases [37, 38]. The Coulomb interaction between massless fermions appears to be unscreened in pristine graphene. At present, it is not clear whether this type of Coulomb interaction would lead to weak correlation in graphene or strongly correlated electronic phases like an insulator $[39,40]$. The long ranged Coulomb interaction in pristine graphene leads to unusual behaviour [41, 42]. The on-site Coulomb interaction plays a crucial role for the understanding of the defect induced magnetisation [43-45].

Earlier, we have reported the study of band gap opening in graphene by a single impurity taking the tight binding model up to the third nearest-neighbour hoppings in the absence of Coulomb interaction [46], in presence of Coulomb interaction [47], electron phonon interaction [48] and bi-layer graphene [49]. In the present brief review, we study the effect of all interactions on the band gap opening of graphene. We propose a tight-binding model Hamiltonian consisting of the site energy of the carbon atoms and nearest- neighbour hopping of $\pi$ electrons of carbon atoms taking into account the substrate effects, Coulomb interaction, electron-phonon interaction effect on monolayer graphene and finally the effect of gate potential on the band gap of bi-layer graphene. The rest of the work is as follows. We describe the tightbinding model Hamiltonian for different types of interaction. We briefly outline the single particle Green's function to calculate correlation functions, physical parameters, quasi-particle energies and electron density of states in the next section. Then we present the results and discussion and finally the conclusion.

\section{Tight-binding model}

The graphene sheet is formed by carbon atoms arranged in a non-Bravais honeycomb lattice with nearest-neighbour C-C distance of $a_{0}=1.43 \AA$ where the lattice constant is $a=\sqrt{3} a_{0}$. The s, $p_{x}$ and $p_{y}$ orbitals hybridise to form $s p^{2}$ bonds leading to high energy sigma bonds. The $p_{z}$ orbitals in graphene form the $\pi$ bond which is responsible for the electronic properties of graphene. The $2 \mathrm{D}$ character of the honeycomb lattice does not allow the overlap of the $p_{z}$ orbital of carbon atom and $\mathrm{s}, p_{x}$ and $p_{y}$ orbitals of its nearest-neighbour carbon atoms. One can construct a simple tight-binding model incorporating only the nearestneighbour hopping of electrons between the adjacent $\mathrm{A}$ and B sub-lattices of honeycomb lattice to study the low energy electron excitations and doping effect in pristine graphene.

Assuming the electron hopping up to the third nearestneighbours, the tight-binding Hamiltonian for electron in graphene is written as

$$
\begin{aligned}
H_{0}= & \sum_{i, \sigma}\left(\epsilon_{a} a_{i, \sigma}^{\dagger} a_{i, \sigma}+\epsilon_{b} b_{i, \sigma}^{\dagger} b_{i, \sigma}\right) \\
& -t_{1} \sum_{<i, j>, \sigma}\left(a_{i, \sigma}^{\dagger} b_{j, \sigma}+b_{j, \sigma}^{\dagger} a_{i, \sigma}\right) \\
& -t_{2} \sum_{\ll i, j \gg, \sigma}\left(a_{i, \sigma}^{\dagger} a_{j, \sigma}+b_{j, \sigma}^{\dagger} b_{i, \sigma}\right) \\
& -t_{3} \sum_{\ll<i, j \gg>, \sigma}\left(a_{i, \sigma}^{\dagger} b_{j, \sigma}+b_{j, \sigma}^{\dagger} a_{i, \sigma}\right)
\end{aligned}
$$

where $a_{i, \sigma}^{\dagger}\left(a_{i, \sigma}\right)$ creates (annihilates) an electron with spin $\sigma$ $(\sigma=\uparrow, \downarrow)$ on site $\overrightarrow{\mathrm{R}}_{\mathrm{i}}$ on sub-lattice A. Similarly, $b_{i, \sigma}^{\dagger}\left(b_{i, \sigma}\right)$ creates (annihilates) an electron on sub-lattice B. Here, $t_{1}(=2.5$ to $3.0 \mathrm{eV})$ is the nearest-neighbour hopping energy, $t_{2}$ with $0.02 t_{1} \leq t_{2} \leq 0.2 t_{1}[36,37]$ is the nextnearest-neighbour hopping energy, $t_{3}$ is the third nearestneighbour hopping integral with $t_{3}=0.024 t_{1} \mathrm{eV}$ and $\varepsilon_{\mathrm{a}}\left(\varepsilon_{\mathrm{b}}\right)$ is the site energy at the sub-lattice site A (B). Further, $\langle i, j\rangle, \ll i, j \gg$, and $\ll\langle i, j\rangle \gg$ stand for nearest, next-nearest and next-next-neighbour hoppings from site $\overrightarrow{\mathrm{R}}_{i}$ to $\overrightarrow{\mathrm{R}}_{j}$. 
The Fourier transformed dispersion $\gamma_{1}(k)$ for the nearestneighbour hopping is

$\gamma_{1}(k)=e^{i k_{x} a_{0}}+2 e^{-\frac{i}{2} k_{x} a_{0}} \cdot \cos \frac{\sqrt{3}}{2} k_{y} a_{0}$

and dispersions for the next-and next-to-next-nearestneighbour hoppings are $\gamma_{2}(k)=\sum_{\delta_{2}} e^{i \vec{k} . \vec{\delta}_{2}} \quad$ and $\gamma_{3}(k)=\sum_{\delta_{3}} e^{i \vec{k} . \vec{\delta}_{3}}$. where $\overrightarrow{\delta_{2}}$ and $\overrightarrow{\delta_{3}}$ are the corresponding lattice vectors. Graphene deposited on silicon dioxide $\left(\mathrm{SiO}_{2}\right)$ substrate can be described by the 2D massless Dirac fermions [3]. On the other hand, graphene deposited on silicon carbide ( $\mathrm{SiC}$ ) substrate can be described by massive 2D Dirac electron. A substrate-induced potential thus generated can break the symmetry of the honeycomb lattice and generate gap in electron system. In this case, the electron interacts with the static potential induced by the substrates and hence A sub-lattice site acquires an energy $+\Delta$ and the B site with energy $-\Delta$ leading to the symmetry breaking between A and B sites. Such a symmetry breaking Hamiltonian is written as

$H_{\mathrm{sub}}=\Delta \sum_{i, \sigma} a_{i, \sigma}^{\dagger} a_{i, \sigma}-\Delta \sum_{i, \sigma} b_{i, \sigma}^{\dagger} b_{i, \sigma}$.

The system exhibits an insulating ground state with a band gap $2 \Delta$, while Fermi level lies in the gap in the undoped system. The Coulomb interaction forbids both the electron occupancies at the same site. The Hubbard interaction representing the two sub-lattices with an effective Coulomb energy $\mathrm{U}$ is written as

$H_{\mathrm{U}}=U \sum_{i}\left[n_{i, \uparrow}^{a} n_{i, \downarrow}^{a}+n_{i, \uparrow}^{b} n_{i, \downarrow}^{b}\right]$

where $n_{i, \uparrow}^{\alpha}\left(n_{i, \downarrow}^{\alpha}\right)$ with $\alpha \in A, B$ sub-lattices, represents the occupation number with up(down) spin. For weak coupling, the Hamiltonian can be decoupled by Hartree-Fock mean-field approximation decoupling scheme, i.e. $U n_{i, \uparrow}^{\alpha} n_{i, \downarrow}^{\alpha} \approx$ $U<n_{i, \uparrow}^{\alpha}>n_{i, \downarrow}^{\alpha}+U<n_{i, \downarrow}^{\alpha}>n_{i, \uparrow}^{\alpha}-U<n_{i, \uparrow}^{\alpha}><n_{i, \downarrow}^{\alpha}>$, where $\alpha \equiv a, b$ corresponding to $\mathrm{A}$ and $\mathrm{B}$ site interactions. The mean-field solutions are taken as $\frac{\left.<n_{i \uparrow \uparrow}^{a}>+<n_{i \uparrow}^{b}\right\rangle}{2}=n$ and $\frac{<n_{i, \uparrow}^{a}>-<n_{i \uparrow}^{b}>}{2}=d$ and this leads to the condition, $\left.<n_{i, \uparrow}^{a}\right\rangle=n+d$ and $\left\langle n_{i, \uparrow}^{b}>=n-d\right.$ where $n$ represents the mean electron occupation and $d$, the deviation from the mean occupation. Similar expression can be formed for the down spin electron. The electron-phonon interaction and lattice vibration are written as

$$
\begin{aligned}
& H_{\mathrm{e}-\mathrm{ph}}=\sum_{\alpha, i, j} f(i-j) n_{i, \sigma}^{\alpha}\left(b_{j}^{\dagger}+b_{j}\right) \\
& H_{\mathrm{p}}=\sum_{j} \omega_{0} b_{j}^{\dagger} b_{j} .
\end{aligned}
$$

Here $H_{\mathrm{e}-\mathrm{ph}}$ describes electron-phonon interaction where the electron density $n_{i, \sigma}^{\alpha}$ at sub-lattice site is coupled to the phonon displacement $u_{j}=b_{j}^{\dagger}+b_{j}$ with phonon creation (annihilation) operator $b_{j}^{\dagger}\left(b_{j}\right)$ at site $j$ and $f(i-j)$ is the electron-phonon coupling. For high frequency phonons, Eq. (5) represents the Holstein interaction. The Hamiltonian $H_{\mathrm{p}}$ represents the free phonon energy with phonon frequency $\omega_{0}$.

\section{Calculation of Green's functions and quasi- particle bands}

The Green's functions for the electrons of A and B sublattices are calculated by Zubarev's Green's function technique [50]. The Zubarev's double time single particle retarded Green's function is defined

$$
\begin{aligned}
G_{\mathrm{r}}\left(t, t^{\prime}\right) & =\ll A(T) ; B\left(t^{\prime}\right) \gg_{\mathrm{r}} \\
& =-i \Theta\left(\mathrm{t}-\mathrm{t}^{\prime}\right)<\left[\mathrm{A}(\mathrm{t}) ; \mathrm{B}\left(\mathrm{t}^{\prime}\right)\right]>
\end{aligned}
$$

where $\langle\ldots\rangle$ indicates the average over a grand canonical ensemble. $A(t)$ and $B\left(t^{\prime}\right)$ are Heisenberg representations of the operators, while $\Theta=1$ for $t>t^{\prime}$ and $\Theta=0$ for $t<t^{\prime}$. Here, $\left[A(t) ; B\left(t^{\prime}\right)\right]$ indicates commutation or anti-commutation depending on Boson or fermions operators. The poles of the Green's functions provide quasi-particle energies.

The correlation function corresponding to Green's function $G_{\mathrm{r}}\left(t, t^{\prime}\right)$ is defined as

$<B\left(t^{\prime}\right) A(t)>=\int_{-\infty}^{\infty} J_{k}(\omega) e^{-i \omega\left(t-t^{\prime}\right)} \mathrm{d} \omega$

where the spectral density function $J_{k}(\omega)$ is written as

$J_{k}(\omega)=i \lim _{\eta \rightarrow 0} \frac{1}{e^{\frac{\omega}{\theta}}+1}\left[G_{\mathrm{r}}(\omega+i \eta)-G_{\mathrm{r}}(\omega-i \eta)\right]$

where $\theta=k_{\mathrm{B}} T$ with $\eta$ as a small spectral width. Other physical parameters can be calculated using the correlation functions of the corresponding Green's functions.

The density of states which is proportional to tunneling conductance is calculated from the imaginary part of Green's function. The electron density of states for the 2D graphene is given by the formula

DOS $=\frac{A}{(2 \pi)^{2}} \iint \overrightarrow{\mathrm{d} k}_{x} \overrightarrow{\mathrm{d} k}_{y} \rho_{k, \sigma}(\omega)$

where $A$ is the area of the honeycomb lattice in real space and spectral function $\rho_{k, \sigma}(\omega)$ is given by 
$\rho_{k, \sigma}(\omega)=-2 \pi \sum_{\sigma}\left[\operatorname{Im}\left(G_{\mathbf{r}}(\omega+i \eta)\right)\right]$

with $\eta$ as a small spectral width. Finally, the occupations and their difference for sub-lattice electrons for different spin orientations are calculated to study the magnetic effect of Coulomb interaction in the gap formation in graphene. All the energy parameters are scaled by the hopping integral $t_{1}$.

\section{Results and discussion}

The band gaps can be induced in graphene near Dirac point by the following techniques.

\section{Effect of electron hopping}

The electron density of states (DOS) and band dispersion are numerically computed [46] and are shown in Figs. 1 and 2. The tight-binding calculations give the first-nearestneighbour hopping integral $t_{1}=2.5-3.0 \mathrm{eV}[30,31]$. In the present calculation, we have taken $\varepsilon_{a}=\varepsilon_{b}=0$,$, t_{2}=-0.12 \mathrm{eV}, t_{3}=-0.068 \mathrm{eV}$. Here, the scaled hopping integrals become $\tilde{t}_{1}=-1, \tilde{t}_{2}=-0.043$ and $\tilde{t}_{3}=-0.024$. The density of states (DOS) for electrons for the graphene is plotted for different band energies (c) (see Fig. 1). The DOS exhibits V-shaped gap structure at K-point (Dirac point) for nearest-neighbour hopping energy $\tilde{t}_{1}=-1$, i.e. DOS shows linear dependence of band energy. When second nearest-neighbour hopping $\left(\tilde{t}_{2}=-0.043\right)$ is included, it still retains the $\mathrm{V}$-shape, but shifts to lower energies becoming asymmetric in nature with respect to Fermi level $\left(\varepsilon_{\mathrm{F}}=0\right)$ at Dirac point (Fig. 1).

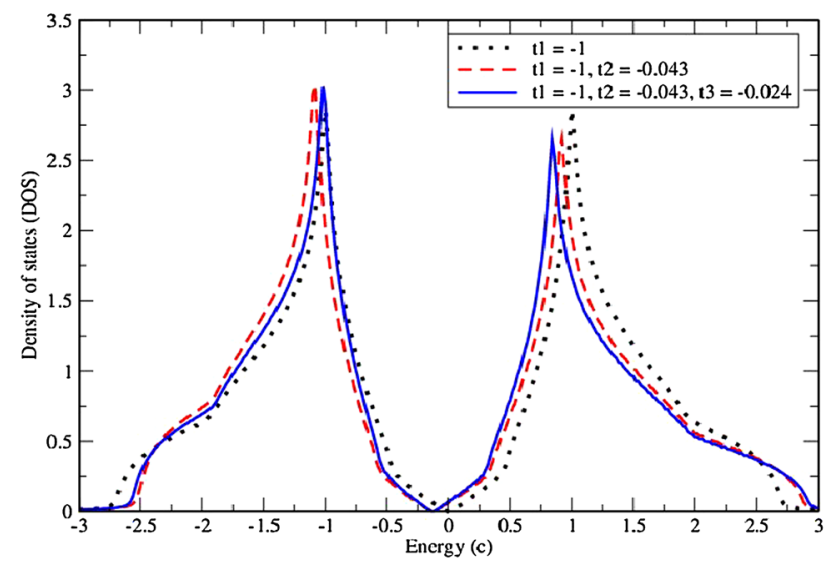

Fig. 1 Plot of the electronic density of states (DOS) of ideal graphene for different band energy with different hopping integrals, $\tilde{t}_{1}=-1$ (solid line) for $\mathrm{NN} ; \tilde{t}_{1}=-1, \tilde{t}_{2}=-0.043$ (dotted line) for $\mathrm{NNN}, \tilde{t}_{1}=-1, \tilde{t}_{2}=-0.043, \tilde{t}_{3}=-0.024$ (dashed line) for NNNN

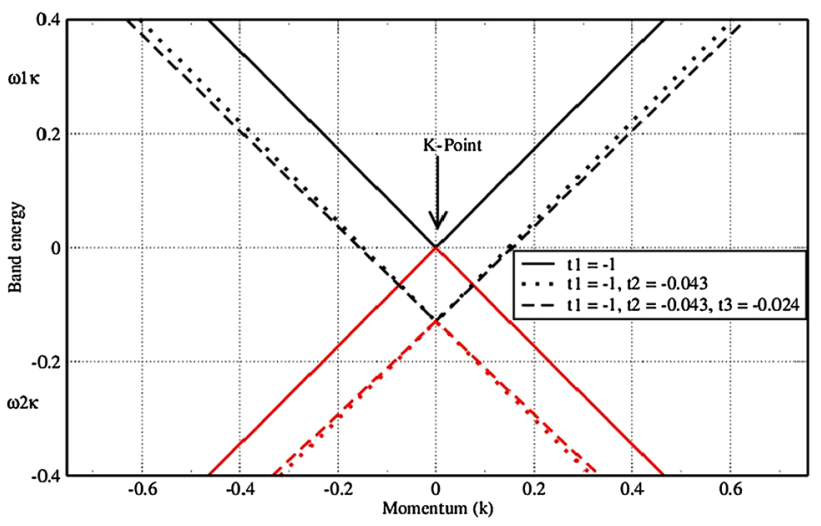

Fig. 2 Plot of the electronic band dispersion of ideal graphene for different band energy with different hopping integrals, $\tilde{t}_{1}=-1$ (solid line) for $\mathrm{NN} ; \tilde{t}_{1}=-1, \tilde{t}_{2}=-0.043$ (dotted line) for $\mathrm{NNN}, \tilde{t}_{1}=-1$, $\tilde{t}_{2}=-0.043, \tilde{t}_{3}=-0.024$ (dashed line) for NNNN

The inclusion of third neighbour interaction induces a gap near K-point and the gap shifts further to lower energies. Figure 2 shows the electron energy dispersion for the graphene and is plotted for different band hopping energy. The energy band dispersion exhibits $\mathrm{V}$-shaped nature at Kpoint (Dirac point) for nearest-neighbour hopping energy $\tilde{t}_{1}=-1$, i.e. band dispersion shows linear dependence of band energy. When second nearest-neighbour hopping $\left(\tilde{t}_{2}=-0.043\right)$ is included, it still retains the V-shape, but shifts to lower energies becoming asymmetric in nature with respect to Fermi level $\left(\varepsilon_{\mathrm{F}}=0\right)$ at Dirac point (Fig. 2). The inclusion of third neighbour interaction induces a gap near $\mathrm{K}$ points and the gap shifts further to lower energies.

\section{Substrate effect}

To study the substrate effect in band gap opening in graphene, we compute DOS and electron band dispersion near Dirac point using the expressions given in Eqs. (1) and (3) of our earlier publications [46] and show the plots in Figs. 3 and 4. Figure 3 shows the DOS for different values of band gaps $d_{1}=0.035-0.107$ developing in graphene sheet due to substrate effect $[9,11,13,18]$ and [32-34]. With the onset of substrate effect, the Dirac point moves towards the valence band occupied by electrons and introduces a band gap below Fermi level $\varepsilon_{\mathrm{F}}=0$. The gap is further enhanced with the increase of $d_{1}$. Figure 4 shows the enhancement of band gap between lower electron occupied valence band and upper hole band due to the increase of $d_{1}$. The middle of the band gap also moves down the Fermi level $\left(\varepsilon_{\mathrm{F}}=0\right)$.

\section{Effect of Coulomb interaction}

The sub-lattice Coulomb interaction is treated within a mean-field approximation. Using the Hamiltonian given in 


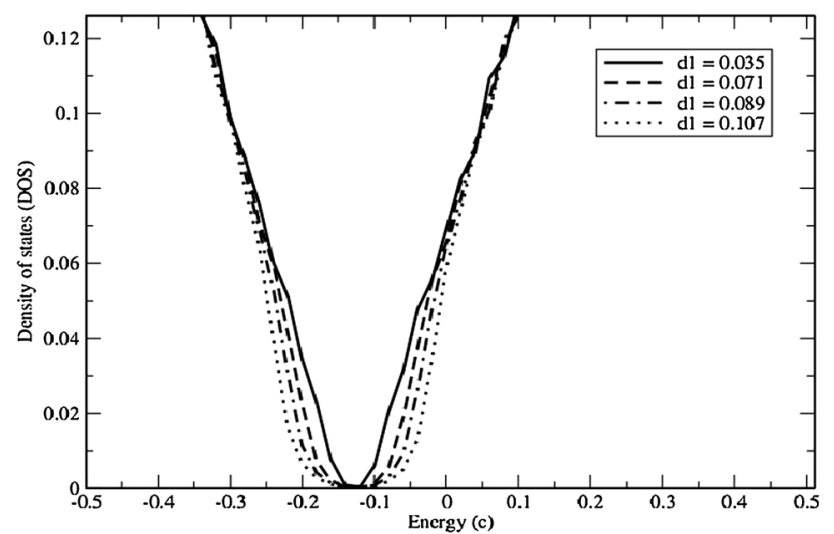

Fig. 3 Plot of the variation of density of states (DOS) with energy (c) for different substrates $d_{1}=0.035,0.071,0.089,0.0107$

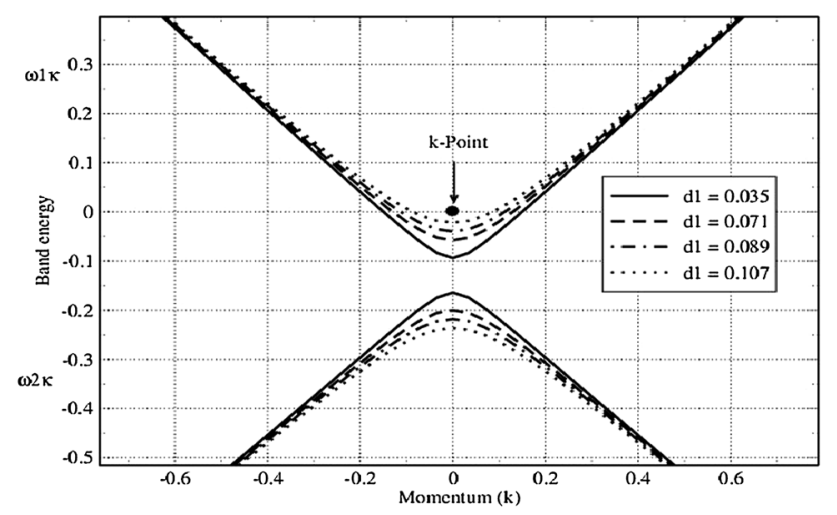

Fig. 4 Plot of the variation of energy band dispersion with momentum $(k)$ for different substrates $d_{1}=0.035,0.071,0.089,0.0107$

Eqs. (1), (3) and (4) of our earlier calculations [47], we calculate the difference $(d)$ in occupation numbers as

$d=\sum_{k} \frac{\bar{\Delta}\left[f\left(\beta \omega_{1 k \uparrow}\right)-f\left(\beta \omega_{2 k \uparrow}\right)\right]}{\left(\omega_{1 k \uparrow}-\omega_{2 k \uparrow}\right)}$

where $f$ is the Fermi distribution function and $\omega_{1 k, \uparrow}$ and $\omega_{2 k, \uparrow}$ are the electron band dispersions. The modified gap $d_{2}(T)=\frac{\tilde{\Delta}}{t_{1}}$ is plotted in Figs. 5, 6 for different Coulomb energies and hopping parameters. The ferromagnetic magnetizations and spin polarizations are reported [51].

The graphene acquires a band gap of $\Delta$ due to substrate effect. The effective band gap then becomes $\bar{\Delta}=\Delta+U \frac{d}{2}$ due to Coulomb interaction between electrons. We scale the physical parameters by nearest-neighbour hopping integral $\left(t_{1}\right)$ and hence the modified gap appears as $d_{2}=d_{1}+u \frac{d}{2}$. The temperature dependent difference $(d)$ in electron occupancies of $\mathrm{A}$ and $\mathrm{B}$ sub-lattices for up spin electrons is computed numerically and self consistently and hence the modified band gap $\left(d_{2}=\frac{\bar{\Delta}}{t_{1}}\right)$ is computed for different Coulomb correlation energies as shown in Fig. 5.

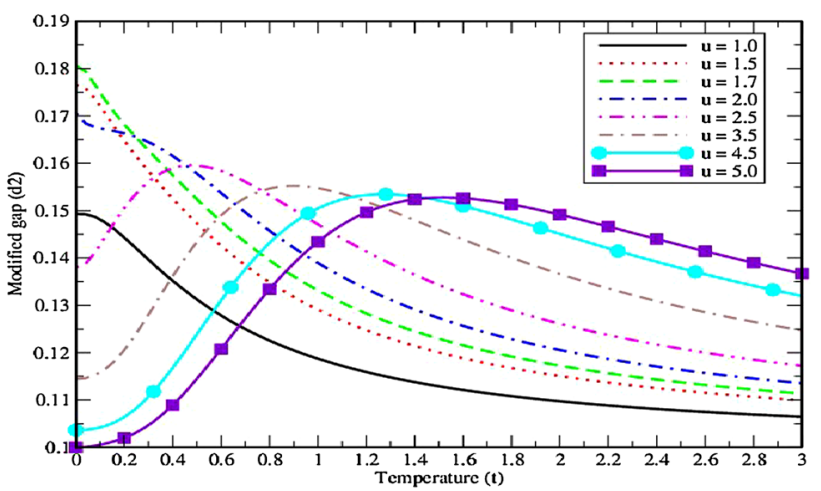

Fig. 5 Plot of the variation of modified band gap $\left(d_{2}\right)$ with temperature $(t)$ for different values of Coulomb energy $u=1.0$, $1.5,1.7,2.0,2.5,3.5,4.5$ and 5.0 for fixed substrate-induced band gap $d_{1}=0.1$

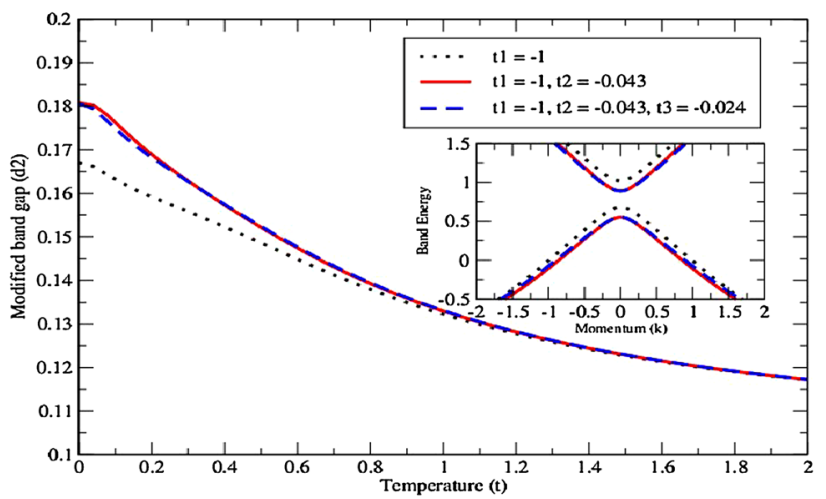

Fig. 6 Plot of the variation of modified band gap $\left(d_{2}\right)$ with temperature $(t)$ at $u=1.7$ for different values of electron hopping $\tilde{t}_{1}=-1, \tilde{t}_{2}=-0.043, \tilde{t}_{3}=-0.024$ for fixed substrate-induced band gap $d_{1}=0.1$. The band dispersions for different hopping are also given in inset of the figure

For lower Coulomb energies, the modified gap $\left(d_{2}\right)$ at $t=0$ gradually increases with increase of $u$ from 1 to 1.7 and attains the maximum of $d_{2}=0.182$. On further increasing to higher Coulomb energies, the modified gap $\left(d_{2}\right)$ at $t=0$ decreases and attains the bare gap $\left(d_{1}\right)$ arising due to only substrate effect for Coulomb energy $u=5$.0. It is observed that the magnetic gap vanishes for $u=5.0$ indicating that $n_{\uparrow}^{a}=n_{\uparrow}^{b}$ in paramagnetic phase. The temperature dependence of modified gap shows that the magnitude of gap for lower ' $u$ ' gradually decreases with temperature. For higher $u$, the modified gap increases with temperature, attains its maximum and then decreases with temperature. However, the maximum of modified gap nearly remains same for higher value of ' $u$ ' at higher temperatures indicating that $d=\left\langle n_{\uparrow}^{a}\right\rangle-\left\langle n_{\uparrow}^{b}\right\rangle$ remains unchanged at very high temperatures and high Coulomb interactions.

Figure 6 shows the effect of different hopping integrals on temperature-dependent modified gap $\left(d_{2}\right)$. In the absence of Coulomb interaction and electron hopping, the 
band gap in graphene is $d_{1}=0.1$ which arises due to substrate effect only. This band gap at temperature $0 \mathrm{~K}$ is enhanced to $d_{2}=0.167$ due to the onset of a critical Coulomb energy $u_{\mathrm{c}}=1.7$ for the nearest-neighbour hopping energy of $t_{1}=-1.0$. This gap at temperature $0 \mathrm{~K}$ is further enhanced to $d_{2}=0.181$ for the same critical Coulomb energy $u_{\mathrm{c}}=1.7$ and for hopping integrals taken up to third nearest neighbours. It appears that the contributions of third nearest-neighbour hopping integrals and beyond have little effect on the band gap. The temperature-dependent modified gap shows that the gap is the highest at very low temperatures and gradually decreases with increase of temperature. This effect of hopping integrals on the modified gap is also seen in the band dispersions shown in the inset of the Fig. 6.

\section{Effect of electron-phonon interaction}

The electrons on graphene sheet interact strongly with the phonons on the polarized surface of substrates. Applying Lang-Firsov canonical transformation [48] to Eqs. (1), (3), (4), (5) and (6) in high frequency limit of localized phonons, we calculate the effective Coulomb energy $\tilde{U}=$ $\left(U-2 \lambda \tilde{t}_{1}\right)$ and effective hopping $\left(\tilde{t}_{1}\right)$ which is a function of phonon frequency $\omega_{0}$, electron-phonon coupling $(\lambda)$ and NN hopping integral $\left(\tilde{t}_{1}\right)$. The temperature-dependent modified gaps are plotted in Figs. 7 and 8.

The effect of Coulomb interaction $(u)$ on the modified gap $d_{2}$ for high phonon frequency $\left(\omega_{0}\right)$ vibration is shown in Fig. 7. For given value of lower Coulomb interaction, the modified gap gradually increases with phonon frequency. With further increase of Coulomb energy, the modified gap gradually increases from $d_{1}=0.1$ and attains maximum value $d_{2}=0.165$ for given Coulomb interaction $u=2.0$. With further increase in Coulomb interaction, the

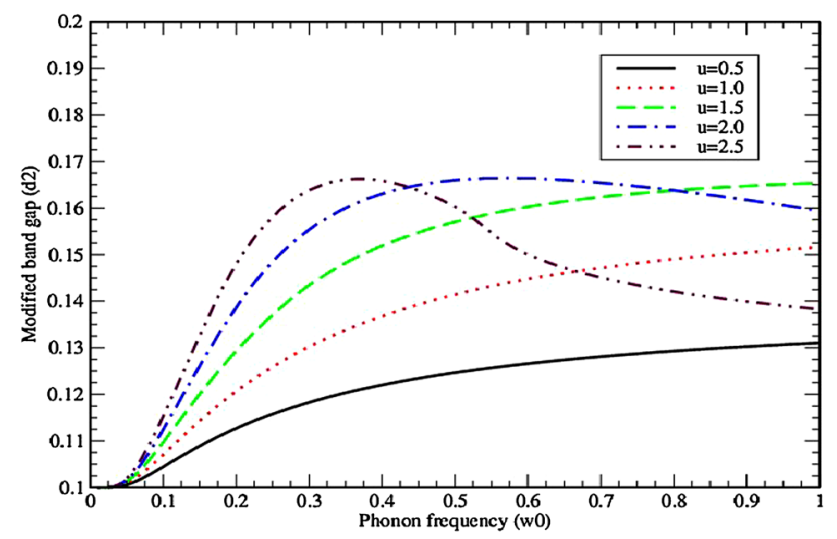

Fig. 7 Plot of the variation of modified band gap $\left(d_{2}\right)$ vs. phonon frequency $\omega_{0}$ for different values of Coulomb potential $u=$ $0.5,1.0,1.5,2.0,2.5$ for fixed electron-phonon coupling constant $a l=0.2$, temperature $(t)=0.01$ and substrate-induced gap $d_{1}=0.1$

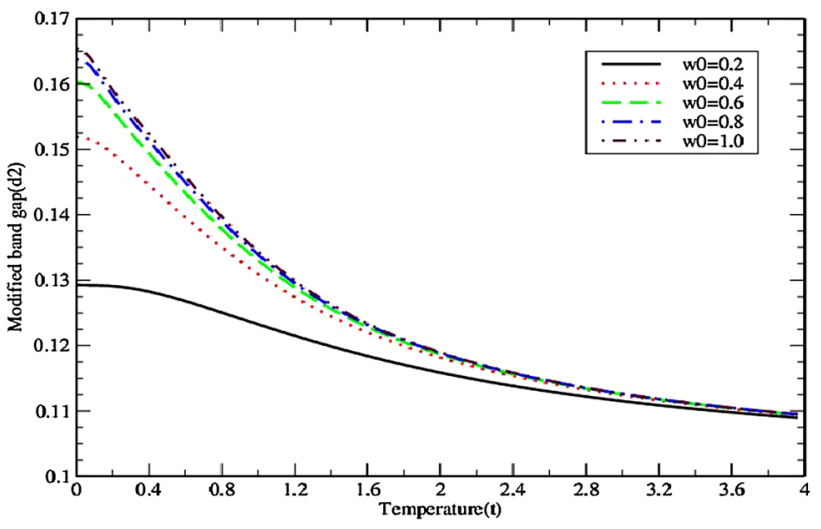

Fig. 8 Plot of the variation of modified band gap $\left(d_{2}\right)$ vs. temperature $(t)$ for different values of phonon frequency $\omega_{0}=$ $0.2,0.4,0.6,0.8,1.0$ for fixed electron-phonon coupling constant $a l=0.2$, Coulomb interaction $(u)=1.7$ and substrate-induced gap $\left(d_{1}\right)=0.1$

modified gap gradually increases with phonon frequency $\omega_{0}$ attains a maximum flat peak with $d_{2}=0.165$ for critical Coulomb interaction $u_{\mathrm{c}}=2.5$ for critical phonon frequency $\omega_{0 \mathrm{c}} \approx 0.35$. Thus, it is clear that modulated gap is maximized for critical Coulomb interaction $u_{\mathrm{c}}=2.5$ and critical phonon frequency $\omega_{0 \mathrm{c}}=0.35$ for given values of electron-phonon coupling $a l=0.2$. The critical Coulomb interaction $U_{\mathrm{C}}=2.5 t_{1}$ for producing maximum modified gap is slightly higher than the critical Coulomb interaction $U_{\mathrm{c}}=u_{\mathrm{c}} t_{1}=1.7 t_{1}$ obtained earlier in the absence of electron-phonon interaction and phonon frequency [47].

The effect of phonon frequency $\left(\omega_{0}\right)$ on gap is shown in Fig. 8. For a given low electron-phonon coupling $a l=0.2$ and relatively low phonon frequency $\omega_{0}=0.2$, the modified gap becomes $d_{2}=0.13$ gap at temperature $t=0$. With increase of phonon vibrational frequency, the modified gap is enhanced to the higher value, i.e. $d_{2}=0.165$ at temperature $t=0$ for vibration frequency $\omega_{0}=1$. However, for a given phonon vibration frequency, the modified gap decreases with temperature and remains nearly constant at higher temperatures. Thus, it is clear that phonon vibration frequency enhances the gap near room temperature.

\section{Effect of bi-layer graphene (BLG)}

Experiments [8, 20, 23] show the evidence of band gap opening in graphene in BLG by gating between two layers. We have proposed model Hamiltonian consisting of intralayer and inter-layer hopping integrals $t_{1}$ and $t_{\perp}$, respectively, in the presence of gating potential $\mathrm{V}[49,52,53]$. The four bands for BLG are written as

$\omega_{\alpha, s,}(k)=-\mu-(-1)^{s} \sqrt{\frac{\left[\frac{V^{2}}{2}+2 \varepsilon_{k}^{2}+\varepsilon_{k, \perp}^{2}-(-1)^{\alpha} \sqrt{R}\right]}{2}}$ 


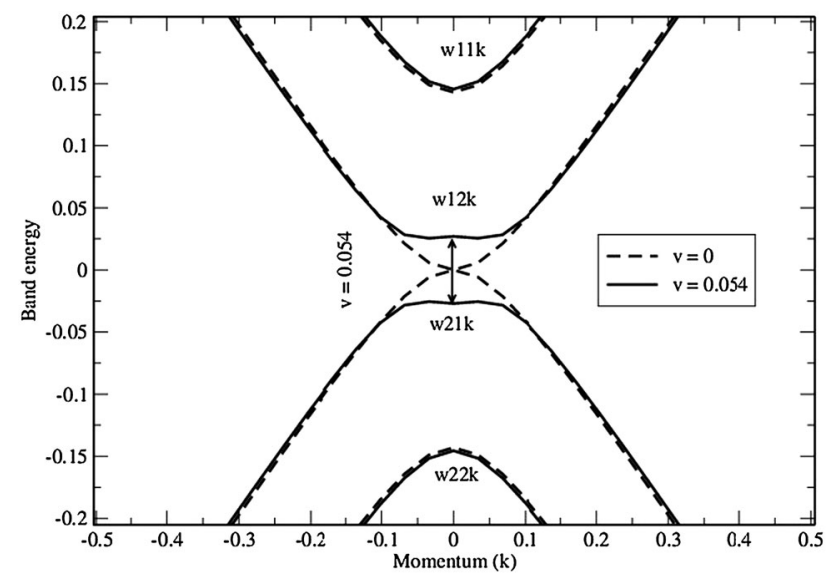

Fig. 9 Plot of energy band dispersions vs. momentum (k) for pristine bi-layer graphene for different values of external biasing $v=0$ (dashed line) and $v=0.054$ (solid line)

$R=\sqrt{\left(V^{2} / 2+2 \varepsilon_{k}^{2}+\varepsilon_{k \perp}^{2}\right)^{2}-4\left(V^{2} / 16+\varepsilon_{k}^{2} V^{2} / 2+\varepsilon_{k \perp}^{2} V^{2} / 4+\varepsilon_{k}^{4}\right)}$

where $s, \alpha=1,2$. The band dispersion is plotted in Fig. 9 which shows four bands $\omega_{\alpha, s k}$ of which $\omega_{11 k}$ and $\omega_{22 k}$ are high energy bands and $\omega_{12 k}$ (conduction) and $\omega_{21 k}$ (valence) bands are the low lying dispersion bands near Dirac point with zero energy. In the absence of gate potential $v=\frac{V}{t_{1}}=0$, low energy bands touch at Dirac point. For low electric potential $(v=0.054)$, the low energy bands show a Mexican hat shape with a gap energy of 0.054 as observed experimentally $[8,23]$.

\section{Conclusions}

The pristine graphene is a gapless semiconductor. It is necessary to induce a band gap in graphene near the Dirac point for its application in several electronic devices. We present here different model calculations for band gap opening in monolayer and bi-layer graphenes taking electron hoppings up to third nearest-neighbours, substrate effect, on-site Coulomb correlation effect and finally electron-phonon interaction along with lattice vibration effect. All the calculations are done using Zubarev's double time single particle Green's function. The expression for modified band gap, dispersions and density of states (DOS) are calculated and computed numerically. The results are discussed varying different model parameters. The DOS exhibits a V-shaped gap near Dirac point with linear energy dependence for nearest-neighbour hopping $t_{1}$. The DOS retains its $\mathrm{V}$-shape for second and third nearest-neighbour hoppings except that the DOS shifts towards low energy valence band. Similar result is concluded from band dispersions for different hopping integrals.
The DOS exhibits wider gap near Dirac point with the increase of band gap arising due to substrate effect. Similar conclusion is derived from the band dispersions also. The substrate-induced gap is modified due to Coulomb interaction energy. The modified gap is enhanced at temperature $T=0^{0} \mathrm{~K}$ with the increase of Coulomb interaction. The effective gap attains its maximum value, $d_{2}=0.182$ at $t=0$ for critical Coulomb interaction $U_{\mathrm{C}}=1.7 t_{1}$. On further increase in the Coulomb energy to $U=5.0 t_{1}$, we observe that the modified gap $\mathrm{d}_{2}=\mathrm{d}_{1}$ indicating that the electron occupancies of both sub-lattices becoming same (i.e. $n_{\uparrow}^{a}=n_{\uparrow}^{b}$ ) in paramagnetic phase. We have calculated the modified band gap taking the electron-phonon interaction between the graphene layer and the substrate in high frequency limit of the localized phonon. In the presence of electron-phonon interaction and phonon vibration, the modified gap attains its maximum value (i.e. $d_{2}=0.165$ ) for a critical Coulomb interaction $U_{\mathrm{c}}=2.5 t_{1}$ which is higher than the critical Coulomb interaction $U_{\mathrm{c}}=1.7 t_{1}$ obtained earlier in the absence of electron-phonon interaction and phonon vibration [47]. Here, we have proposed tight-binding model calculation for AB-stacked bi-layer graphene taking intra-layer and inter-layer hopping in the presence of transverse gating potential. We have obtained four quasi-particle bands of which the inner two bands touch each other at Dirac point. A band gap opens near Dirac point due to application of the gating potential. These two inner bands exhibit Mexican hat shape for gating potential $V=0.054 t_{1}$. Since we have considered dispersion for full Brillouin zone in both the layers, we can take any gating potential higher than the gating potential $V=$ $0.054 t_{1}$ applied to bi-layer systems experimentally $[8,23]$.

Acknowledgements The authors gracefully acknowledge the research facilities offered by the Institute of Physics, Bhubaneswar, India.

Open Access This article is distributed under the terms of the Creative Commons Attribution 4.0 International License (http://crea tivecommons.org/licenses/by/4.0/), which permits unrestricted use, distribution, and reproduction in any medium, provided you give appropriate credit to the original author(s) and the source, provide a link to the Creative Commons license, and indicate if changes were made.

\section{References}

1. Novoselov, K.S., Geim, A.K., et al.: Electric field effect in atomically thin carbon films. Science. 306, 666 (2004)

2. Novoselov, K.S., Geim, A.K., Morozov, S.V., Jiang, D., et al.: Two-dimensional gas of massless Dirac fermions in graphene. Nature 438, 197 (2005)

3. Wallace, P.R.: The Band Theory of Graphite. Phys. Rev. 71, 622 (1947)

4. Miller, D.L., Kubista, K.D., et al.: Observing the Quantization of ZeroMass Carriers in Graphene. Science. 324, 924 (2009) 
5. Stander, N., Huard, B., Goldhaber-Gordon, D.: Evidence of Klein tunneling in graphene pn junctions. Phys. Rev. Lett. 102, 026807 (2009)

6. Bolotin, K.I., Sikes, K.J., Hone, J., et al.: Temperature-Dependent Transport in Suspended Graphene. Phys. Rev. Lett. 101, 096802 (2008)

7. Han, M.Y., Özyilmaz, B., et al.: Energy Band-Gap Engineering of Graphene Nanoribbons. Phys. Rev. Lett. 98, 206805 (2007)

8. McCann, E., Falko, V.I.: Landau level degeneracy and quantum Hall effect in a graphite bilayer. Phys. Rev. Lett. 96, 086805 (2006)

9. Giovannetti, G., Khomyakov, P.A., et al.: Substrate-induced band gap in graphene on hexagonal boron nitride: $\mathrm{Ab}$ initio density functional calculations. Phys. Rev. B. 76, 073103 (2007)

10. Jung, J., DaSilva, A.M., MacDonald, A.H., Adam, S.: Origin of band gaps in graphene on hexagonal boron nitride. Nat. Commun. 6, 6308 (2015)

11. Zhou, S.Y., Gweon, G.-H., Fedorov, A.V., et al.: Substrate-induced bandgap opening in epitaxial graphene. Nat. Mater. 6, 770 (2007)

12. Nevius, M.S., Conrad, M., Wang, F., Celis, A., Nair, M.N., Taleb-Ibrahimi, A., Tejeda, A., Conrad, E.H.: Semiconducting Graphene from Highly Ordered Substrate Interactions. Phys. Rev. Lett. 115, 136802 (2015)

13. Varchon, F., Feng, R., Hass, J., et al.: Electronic Structure of Epitaxial Graphene Layers on SiC: Effect of the Substrate. Phys. Rev. Lett. 99, 126805 (2007)

14. Mattausch, A., Pankratov, O.: Ab Initio Study of Graphene on SiC. Phys. Rev. Lett. 99, 076802 (2007)

15. Emtsev, K.V., Speck, F., Seyller, Th, Ley, L.: Interaction, growth, and ordering of epitaxial graphene on $\mathrm{SiC}\{0001\}$ surfaces: A comparative photoelectron spectroscopy study. Phys. Rev. B. 77, 155303 (2008)

16. Varchon, F., Mallet, P., Veuillen, J.-Y., et al.: Ripples in epitaxial graphene on the Si-terminated $\mathrm{SiC}(0001)$ surface. Phys. Rev. B. 77, 235412 (2008)

17. Varchon, F., Mallet, P., Veuillen, J.-Y., et al.: Why Multilayer Graphene on $4 \mathrm{H}-\mathrm{SiC}(000-1)$ Behaves Like a Single Sheet of Graphene. Phys. Rev. Lett. 100, 125504 (2008)

18. Fratini, S., Guinea, F.: Substrate-limited electron dynamics in graphene. Phys. Rev. B. 77, 195415 (2008)

19. Zhao, J., Wenbin, H., Li, H., Ji, M., Zhao, C., Wang, Z., Haiqing, H.: One-step green synthesis of a ruthenium/graphene composite as a highly efficient catalyst. RSC Adv. 5, 7679 (2015)

20. McCann, D.S.L., Abergel, V.I.: Electrons in bilayer graphene. Falko. Solid State Commun. 143, 110 (2007)

21. Zhang, Y., Tang, T.-T., Girit, C., Hao, Z., et al.: Direct observation of a widely tunable bandgap in bilayer graphene. Nature. 459, 820 (2009)

22. Abergel, D.S.L.: Mucha-Kruczyński, Marcin.: Infrared absorption of closely aligned heterostructures of monolayer and bilayer graphene with hexagonal boron nitride. Phys. Rev. B. 92, 115430 (2015)

23. Ohta, T., Bostwick, A., Seyller, T., Horn, K., Roteberg, E.: Controlling the electronic structure of bilayer graphene. Science. 313, 951 (2006)

24. Hague, J.P.: Tunable graphene band gaps from superstrate-mediated interactions. Phys. Rev. B. 84, 155438 (2011)

25. Hague, J.P.: Polarons in highly doped atomically thin graphitic materials. Phys. Rev. B. 86, 064302 (2012)

26. Halasall, M.P., Ferrari, A.C., et al.: Control of graphene's properties by reversible hydrogenation: evidence for graphane. Science. 323, 610 (2009)

27. Eklund, P.C., Sofo, J.O., Zhu, J.: Reversible fluorination of graphene: Evidence of a two-dimensional wide bandgap semiconductor. Phys. Rev. B. 81, 205435 (2010)
28. Lin, X., Yang, X., Hakro, A.A., Hasan, T., Hao, R., Zhang, B., Chen, H.: Ab initio optical study of graphene on hexagonal boron nitride and fluorographene substrates. J. Mater. Chem. C. 1, 1618 (2013)

29. Song, L., Ci, L., Lu, H., et al.: Large scale growth and characterization of atomic hexagonal boron nitride layers. Nano Lett. 10, 3209 (2010)

30. Wehling, T.O., Şaşığlu, E., Friedrich, C., et al.: Strength of Effective Coulomb Interactions in Graphene and Graphite. Phys. Rev. Lett. 106, 236805 (2011)

31. Kotov, Valeri N., Uchoa, Bruno, et al.: Electron-Electron Interactions in Graphene: Current Status and Perspectives. Rev. Mod. Phys. 84, 1067 (2012)

32. Hansmann, P., Ayral, T., Vaugier, L., et al.: Long-Range Coulomb Interactions in Surface Systems: A First-Principles Description within Self-Consistently Combined GW and Dynamical Mean-Field Theory. Phys. Rev. Lett. 101, 166401 (2013)

33. Pariser, R., Parr, G.: A Semi-Empirical Theory of the Electronic Spectra and Electronic Structure of Complex Unsaturated Molecules. J. Chem. Phys. 21, 767 (1953)

34. Soos, Z.G., Ramasesha, S., Galvo, D.S.: Band to correlated crossover in alternating Hubbard and Pariser-Parr-Pople chains: Nature of the lowest singlet excitation of conjugated polymers. Phys. Rev. Lett. 71, 1609 (1993)

35. Friend, R.H., Gymer, R.W., Holmes, A.B., et al.: Electroluminescence in conjugated polymers. Nature. 397, 121 (1999)

36. Schuler, M., Rosner, M., Wehling, T.O., et al.: Optimal Hubbard Models for Materials with Nonlocal Coulomb Interactions: Graphene, Silicene, and Benzene. Phys. Rev. Lett. 111, 036601 (2013)

37. Meng, Z.Y., Lang, T.C., Wessel, S., Assaad, F.F., Muramatsu, A.: Quantum spin liquid emerging in two-dimensional correlated Dirac fermions. Nature. 464, 847 (2010)

38. Osofsky, M.S., Hernández, S.C., Nath, A., Wheeler, V.D., Walton, S.G., Gaskill, D.K.: Functionalized graphene as a model system for the two-dimensional metal-insulator transition. Sci. Rep. 6, 19939 (2016)

39. Igor, F.: Herbut.: Interactions and Phase Transitions on Graphene's Honeycomb Lattice. Phys. Rev. Lett. 97, 146401 (2006)

40. Drut, J.E., Lähde, T.A.: Is Graphene in Vacuum an Insulator? Phys. Rev. Lett. 102, 026802 (2009)

41. Castro Neto, A.H., Guinea, F., et al.: The electronic properties of graphene. Rev. Mod. Phys. 81, 109 (2009)

42. Das Sarma, S., Shaffique, A., et al.: Electronic transport in twodimensional graphene. Rev. Mod. Phys. 83, 407 (2011)

43. Parr, R.G., Craig, D.P., Ross, I.G.: Molecular orbstal calculations of the lower excited electronic levels of benzene, configuration interaction included. J. Chem. Phys. 18, 1561 (1950)

44. Wang, Y.F., Singh, S.B., Limaye, M.V., Shao, Y.C., Hsieh, S.H., Chen, L.Y., Hsueh, H.C., Wang, H.T., Chiou, J.W., Yeh, Y.C., Chen, C.W., Chen, C.H., Ray, S.C., Wang, J., Pong, W.F., Takagi, Y., Ohigashi, T., Yokoyama, T., Kosugi, N.: Visualizing chemical states and defects induced magnetism of graphene oxide by spatially-resolved-X-ray microscopy and spectroscopy. Sci. Rep. 5, 15439 (2015)

45. Midtvedt, D., Alexander, C.: Strain-displacement relations for strain engineering in single-layer $2 \mathrm{~d}$ materials. J. Phys. Condens. Matter. 28, 045302 (2016)

46. Sahu, S., Rout, G.C.: Study of Band Gap Opening in Graphene by Impurity and Substrate-Mediated Interactions. Adv. Sci. Lett. 20, 834 (2014)

47. Sahu, S., Rout, G.C.: Model study of the effect of coulomb interaction on band gap of graphene-on-substrates. Phys. B. 461, 49 (2015)

48. Sahu, S., Parashar, S.K.S., Rout, G.C.: Model study of band gap opening in graphene by electron-electron and electron-phonon 
interaction in high frequency range. Adv. Sci. Lett. 22, 331 (2016)

49. Sahu, S., Parashar, S.K.S., Rout, G.C.: Theoretical study of band gap opening in $\mathrm{AB}$-stacked bi-layer graphene by impurity and electric field effects. Mat. Today Proc. 3, 39 (2016)

50. Zubarev, D.N.: Double-time green functions in statistical physics. Sov. Phys. Usp. 3, 320 (1960)

51. Sahu, S., Rout, G.C.: Tight-binding model study of substrate induced pseudo-spin polarization and magnetism in mono-layer graphene. J. Magn. Magn. Mater. 407, 396 (2016)
52. Rout, G.C., Sahu, S., Panda, S.K.: Effect of impurity doping on tunnelling conductance in $\mathrm{AB}$-stacked bi-layer graphene: A tightbinding study. AIP Conf. Proc. 1724, 020125 (2016)

53. Sahu, S., Parashar, S.K.S., Rout, G.C.: Microscopic theoretical model study of band gap opening in AA-stacked bi-layer graphene. AIP Conf. Proc. 1728, 020037 (2016) 